\title{
PROPAGATION OF FLAME FRONTS
}

\author{
N. F. SMYTH ${ }^{1}$ \\ (Received 19 January 1989; revised 20 July 1989))
}

\begin{abstract}
The propagation of a flame front in a combusting gas is considered in the limit in which the width of the reaction-zone is small compared with some overall flow dimension. In this approximation, the front propagates along its normals at a speed dependent on the local curvature of the front and is governed by a nonlinear equivalent of the geometric optics equations. Some exact solutions of this equation are found and a numerical scheme is developed to solve the equation for more complicated geometries.
\end{abstract}

\section{Introduction}

In linear wave theory, geometric optics provides a simple (and accurate) method of obtaining approximate solutions of wave propagation problems when the wave field is slowly varying or when the motion of a front is being considered. In this approximation, a wave front is propagated along its normals at the local wave speed. The question naturally arises as to whether geometric optics can be extended to nonlinear wave propagation problems. In what was called geometrical shock dynamics, Whitham [7], [8], applied the ideas of geometric optics to the propagation of shock waves. In this theory, a shock is propagated along its normals at a local Mach number $M$ which depends on the local area $A$ of the shock, these being related by the $A-M$ relation

$$
A / A_{0}=f(M) / f\left(M_{0}\right)
$$

\footnotetext{
'Department of Mathematics, University of Wollongong, P.O. Box 1144, Wollongong, N.S.W. 2500, Australia.

(C) Copyright Australian Mathematical Society 1990, Serial-fee code 0334-2700/90
} 
where $M_{0}$ and $A_{0}$ are the initial Mach number and area respectively and $f$ is some function whose details need not be given here. The equations of geometrical shock dynamics form a second order system of nonlinear hyperbolic equations and a number of solutions were found by Whitham [7], [8], [9]. These solutions were found to agree very well with both exact solutions of the gas equations and experimental results.

Since geometrical shock dynamics involves propagating a shock alôñ its normals at a Mach number determined by (1.1), it is a very fast method of propagating shocks numerically. Henshaw, Smyth and Schwendeman [4] developed such a numerical method and used it to study a variety of shock propagation problems. Good agreement was found between their results and both analytical and experimental results.

The application of geometric optics to nonlinear problems is not limited to geometrical shock dynamics and can be applied to many situations in which the propagation of a front is involved. For example, nonlinear geometric optics was developed for soliton propagation problems by Miles [5] and for the propagation of phase change fronts by Ben-Jacob et al [2]. Stewart and Bdzil [6] considered an unsteady flame propagating in a polytropic, combusting gas. On assuming that the reaction-zone thickness is small compared with some overall dimension of the flow, they showed that the flame front propagates along its normals with a local speed $V$ given by

$$
V=D_{C J}-a K \text {, }
$$

where $K$ is the curvature of the front, $D_{C J}$ is the steady one-dimensional Chapman-Jouguet velocity and $a$ is a constant.

In the present work, exact solutions of the equations of motion of the flame front obtained from (1.2) will be found for a flame front propagating in a onedimensional duct and a cylindrical tube. Also, a numerical scheme similar to that of Henshaw, Smyth and Schwendeman [4], but using the normal velocity relation (1.2), will be discussed and used to study the propagation of a flame front in a duct with a hump and in a duct with a $90^{\circ}$ circular bend.

\section{Governing equations}

In the present section, the derivation by Stewart and Bdzil [6] of the normal velocity relation (1.2) from the gas equations will be summarised. Consider an unsteady detonation wave propagating in a polytropic gas filling some tube. Behind this front, there is reacting gas and in order to construct an asymptotic theory, we shall assume that the reaction-zone thickness is small compared to some overall dimension of the flow. We shall further assume that 
the reaction-zone is fully resolved. We shall use a coordinate system moving with the steady detonation velocity $D$, with the $z$ coordinate oriented along the tube. The equations governing the flow of the reacting gas are then

$$
\begin{gathered}
\frac{\partial H}{\partial t}+\underline{u} \cdot \underline{\nabla} H=\frac{1}{\rho} \frac{\partial p}{\partial t} \\
\frac{\partial \underline{\omega}}{\partial t}+\underline{u} \cdot \underline{\nabla \omega}=\underline{\omega} \cdot \underline{\nabla u}+\rho^{-2} \underline{\nabla} \rho \times \underline{\nabla p} \\
\frac{\partial}{\partial t}\left(-\frac{p}{\rho}+\frac{1}{2}|\underline{\nabla} u|^{2}\right)+\underline{u} \cdot \underline{\nabla}\left(\frac{1}{2}|\underline{\nabla} u|^{2}\right)-\frac{\gamma p}{\rho} \underline{\nabla} \cdot \underline{u}=-(\gamma-1) q R \\
\frac{\partial \lambda}{\partial t}+\underline{u} \cdot \underline{\nabla} \lambda=R \equiv k f(\lambda),
\end{gathered}
$$

where

$$
H=\frac{1}{2}|\underline{\nabla} u|^{2}+p / \rho+E .
$$

These equations form an equivalent system to the standard Euler equations. Here $\underline{u}, \underline{\omega}, \rho, p, E$ and $\lambda$ are the fluid velocity relative to the moving frame, vorticity $(=\underline{\nabla} \times \underline{u})$, density, pressure, specific internal energy and reaction progress variable respectively. The progress variable varies between zero and one, being zero when the gas is unreacted and one when it is fully reacted. The reaction rate is denoted by $R$ and the parameter $k$ is a rate multiplier whose reciprocal is a characteristic reaction time. The parameter $\gamma$ is the specific heat ratio for the gas. For a polytropic gas,

$$
E=(\gamma-1)^{-1} p / \rho-q \lambda,
$$

where $q$ is the specific heat of reaction of the gas.

The reaction-zone is preceded by a shock, which we shall assume is strong (i.e. has Mach number $>>1$ ). Let us denote the position of this leading shock by $z=z_{s}$ and denote the state immediately behind the shock by a subscript $s$. Then the jump conditions for a strong shock (Steward and Bdzil [6]) give the boundary conditions

$$
\begin{gathered}
\rho_{s} / \rho_{0}=(\gamma+1) /(\gamma-1), \\
\partial u_{s} / \partial z=-\left\{D\left[(\gamma-1) /(\gamma+1)+\left|\underline{\nabla} z_{S}\right|^{2}\right]-2 /(\gamma+1) \partial z_{S} / \partial t\right\} / F, \\
\underline{\nabla} u_{S}=-\frac{2}{(\gamma+1) F}\left(D+\frac{\partial z_{S}}{\partial t}\right) \underline{\nabla} z_{S}, \\
p_{S}=\frac{2 \rho_{0}}{(\gamma+1) F}\left(D+\frac{\partial z_{S}}{\partial t}\right)^{2},
\end{gathered}
$$

for the reaction-zone, where

$$
F=\left[1+\left|\underline{\nabla} z_{S}\right|^{2}\right]
$$

and $\rho_{0}$ is the density of the quiescent gas ahead of the shock. 
If we define $\delta$ to be the ratio of a length characteristic of the reaction-zone to a length characteristic of the overall flow, then by assumption, $\delta$ is small and serves as a basic perturbation parameter. By solving (2.1) to (2.5) using a perturbation series in $\delta$, Stewart and Bdzil [6] found that the propagation of the flame front is governed by the parabolic equation

$$
\partial z_{S} / \partial T=a \nabla^{2} z_{S}+\frac{1}{2} D_{C I} ! \underline{\nabla} z_{S} !^{2}-D_{2},
$$

where $\underline{\nabla}$ is the scaled gradient operator in the plane tangent to the direction of steady propagation, the derivatives being with respect to the new scaled coordinate

$$
\underline{\zeta}=\delta \underline{x} .
$$

This new variable is a boundary layer coordinate for the (thin) reaction-zone. Furthermore,

$$
\begin{gathered}
T=\delta^{2} t, \\
D=D_{C J}+\delta^{2} D_{2}+\ldots, \\
a=\gamma(\gamma+1) G,
\end{gathered}
$$

where

$$
G=\frac{2 \gamma D_{C J}^{2}}{k(\gamma+1)^{3}} \int_{0}^{1} \frac{\theta(1+\theta)^{2} d \theta}{f(\lambda(\theta))} .
$$

The term $D_{C J}$ is the one-dimensional steady Chapman-Jouguet detonation velocity, given by

$$
D_{C J}=\left[2 q\left(\gamma^{2}-1\right)\right]^{1 / 2}
$$

and $D_{2}$ is the (negative) correction to this velocity due to the effect of the curved front. The parameter $a$ is a measure of the amount of heat released by the reaction.

To complete the description of the flame front, boundary conditions are needed for equation (2.6). The analysis of the boundary layer at the tube wall is a difficult and, as yet, incompletely solved problem. However, work by Bdzil [1] for steady detonation shows that if the time dependence of the flow in the boundary layer occurs on the slow time scale $T=\delta^{2} t$, then the boundary condition

$$
\underline{n} \cdot \underline{\nabla} z_{S}=-(\gamma-1) L / 2
$$

holds at the tube wall, where $\underline{n}$ is the normal to the tube wall and $L$ is an $0(1)$ constant which accounts for the adjustment in the streamline curvature between the interior flow and the flow at the tube wall. This boundary condition will be the one used in the present work. 
Equation (2.6) together with the boundary condition (2.13) can be set into non-dimensional form by introducing the new variables

$$
\begin{gathered}
Z=\frac{D_{C J}}{2 a} z_{S} \\
\underline{\xi}=\left(-\frac{D_{2} D_{C J}}{2 a}\right)^{1 / 2} \zeta \\
\tau=\left(-\frac{D_{C J} D_{2}}{2 a}\right) T \\
W=\frac{1}{2}\left(-\frac{D_{C J}}{2 D_{2}}\right)^{1 / 2}(\gamma-1) L .
\end{gathered}
$$

With these new variables, (2.6) becomes

$$
\partial Z / \partial \tau=\nabla^{2} Z+|\underline{\nabla} Z|^{2}+1
$$

together with the boundary condition

$$
\underline{n} \cdot \nabla Z=-W
$$

on the tube walls. Here $\underline{\nabla}$ is now the gradient operator for derivatives with respect to $\underline{\xi}$. This equation is a Ricatti-type equation in $\underline{\nabla Z}$ and can be transformed to a linear parabolic equation using the transformation

$$
Z=\log \psi
$$

Equation (2.15) together with boundary condition (2.16) then become

$$
\partial \psi / \partial \tau=\nabla^{2} \psi+\psi
$$

with

$$
\underline{n} \cdot \underline{\nabla} \psi+W \psi=0
$$

on the walls. Since (2.18) is a linear equation, it can be solved using standard techniques. As yet, $D_{2}$, the correction to the steady one-dimensional Chapman-Jouguet velocity has not been determined. This quantity is determined from the condition that $(2.18)$ has a steady solution. Some exact solutions of (2.18) and (2.19) will be found in the next section.

The original dimensional flame front equation (2.6) has a simple physical interpretation. From Steward and Bdzil [6], the propagation velocity of the flame front along its normal, $V$, is given by

$$
V=D_{C J}+\delta^{2}\left[\partial z_{S} / \partial T+D_{2}-\frac{1}{2} D_{C J}\left|\underline{\nabla} z_{S}\right|^{2}\right] \text {. }
$$

Also the sum of the principal curvatures of the front is

$$
K=-\delta^{2} \nabla^{2} z_{S}
$$


Hence from (2.6),

$$
V=D_{C J}-a K \text {. }
$$

Therefore the front propagates such that its normal velocity is equal to the steady Chapman-Jouguet velocity minus a constant times curvature. This simple interpretation is the basis of the numerical solution of (2.6) and (2.13) in section 4.

The above asymptotic analysis breaks down in the su-called simpie depeletion limit in which the length of the reaction zone becomes large. For example, if the rate law

$$
f(\lambda)=(1-\lambda)^{\nu}, 0 \leq \nu<1
$$

is assumed, simple depletion occurs in the limit $\nu \rightarrow 1$. In this limit, $a \rightarrow \infty$ as the integral (2.11) does not converge. By separately analysing this limit, Stewart and Bdzil [6] showed that

$$
V=D_{C J}+\beta K \log K+2 \beta K\left[\log \left(\beta / D_{C J}\right)-3\right],
$$

where

$$
\beta=\left[\gamma D_{C J} /(\gamma+1)\right]^{2} k^{-1} .
$$

As the simple depletion limit will not be considered further here, the front velocity relation (2.24) will not be used in this work.

\section{Analytical solutions}

It was shown in the previous section that the propagation of a flame front in a tube is governed by the linear parabolic equation (2.18) with boundary condition (2.19). For simple geometries, this equation can be solved using either separation or variables of Laplace transforms in $\tau$. We shall now consider the two specific examples of a one dimensional straight duct and a cylindrical tube. For simplicity, we shall consider the initial condition

$$
Z=0 \text { at } \tau=0 \text {, }
$$

so that

$$
\psi=1 \text { at } \tau=0 .
$$

Let us first consider a flame front in a one dimensional duct, so that $\psi=$ $\psi(\xi, \tau)$ where $\xi$ is the cross coordinate. Let us take the walls of the duct to be at $\xi= \pm b$. It can easily be found using separation of variables that equation (2.18), together with boundary condition (2.19) and initial condition (3.2), has the solution

$$
\psi=\sum_{n=0}^{\infty} \frac{2 \sin \sqrt{1+\lambda_{n}} b}{b \sqrt{1+\lambda_{n}}} e^{-\lambda_{n} \tau} \cos \sqrt{1+\lambda_{n}} \xi
$$


where $\lambda_{n}$ is a solution of

$$
\tan \sqrt{1+\lambda_{n}} b=W / \sqrt{1+\lambda_{n}}
$$

We still have not fully determined the solution of (2.18) as $D_{2}$ has not yet been determined. This quantity is determined by the requirement that $(2.18)$ has a steady state solution. For a steady solution to exist, we require $\lambda_{0}=0$, so that from (3.4),

$$
W=\tan b,
$$

which gives, on using (2.14)

$$
\left(-\frac{D_{C J}}{2 D_{2}}\right)^{1 / 2}(\gamma-1) L=2 \tan \left[\zeta_{0}\left(-\frac{D_{2} D_{C J}}{2 a}\right)^{1 / 2}\right],
$$

where the dimensional position of the walls is at $\zeta= \pm \zeta_{0}$. This expression (implicitly) determines $D_{2}$. The steady part $(n=0)$ of the solution (3.3) was also found in Stewart and Bdzil [6].

The propagation of a flame front in a cylindrical tube can similarly be determined. In this case, the solution is circularly symmetric with $\psi=\psi(\rho, \tau)$, where $\rho$ is the radial coordinate for cylindrical polar coordinates in $\xi$. In the tube has radius $\rho=b$, then it can be found that (2.18) together with (2.19) and (3.2) has the solution

$$
\psi=\frac{2 W}{b\left(1+W^{2}\right)} \frac{J_{0}(\rho)}{J_{0}(b)}+\sum_{n=1}^{\infty} \frac{2 W}{b\left(1+\lambda_{n}+W^{2}\right)} \frac{J_{0}\left(\sqrt{1+\lambda_{n}} \rho\right)}{J_{0}\left(\sqrt{1+\lambda_{n}} b\right)} e^{-\lambda_{n} \tau},
$$

where

$$
\sqrt{1+\lambda_{n}} J_{1}\left(\sqrt{1+\lambda_{n}} b\right)=W J_{0}\left(\sqrt{1+\lambda_{n}} b\right)
$$

and

$$
W=J_{1}(b) / J_{0}(b) \text {. }
$$

As for the planar case, condition (3.9) was determined by requiring there to be a steady state solution of (2.18). Using the non-dimensionalisation (2.14), $D_{2}$ can be determined from (3.9).

In a similar manner to the above cases, further analytical solutions of (2.18) for simple geometries can be found. In the next section, we shall consider numerical solutions of (2.6).

When the flame front has reached a steady state and is propagating at a constant velocity, we see from (2.18) and (2.19) that it is governed by

$$
\nabla^{2} \psi+\psi=0 \text { in } \Sigma
$$

with

$$
\underline{n} \cdot \underline{\nabla} \psi+W \psi=0 \text { on } \partial \Sigma
$$


(a)

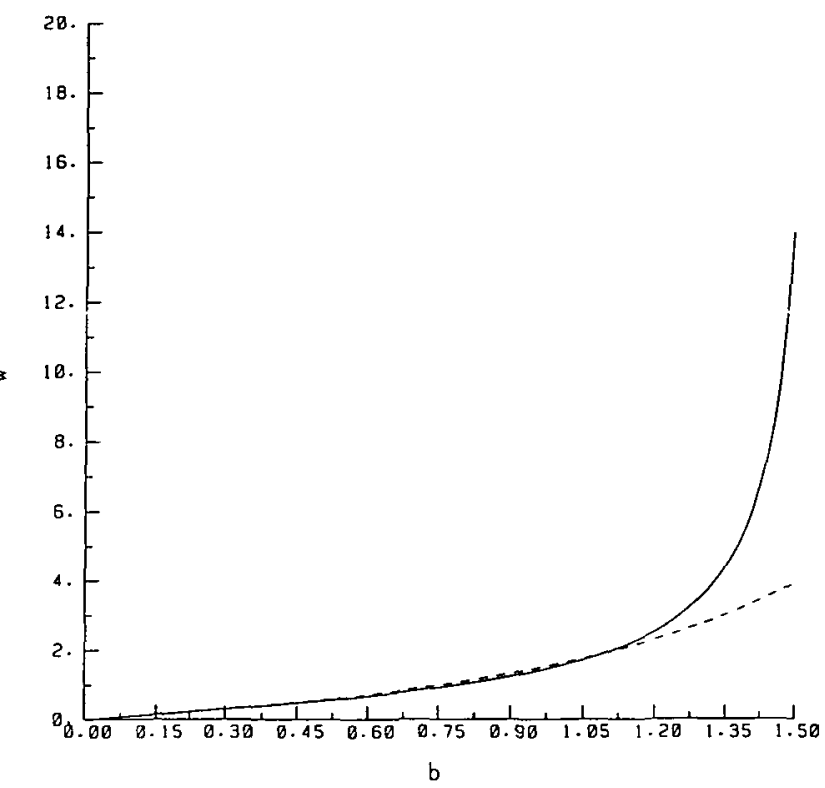

(b)

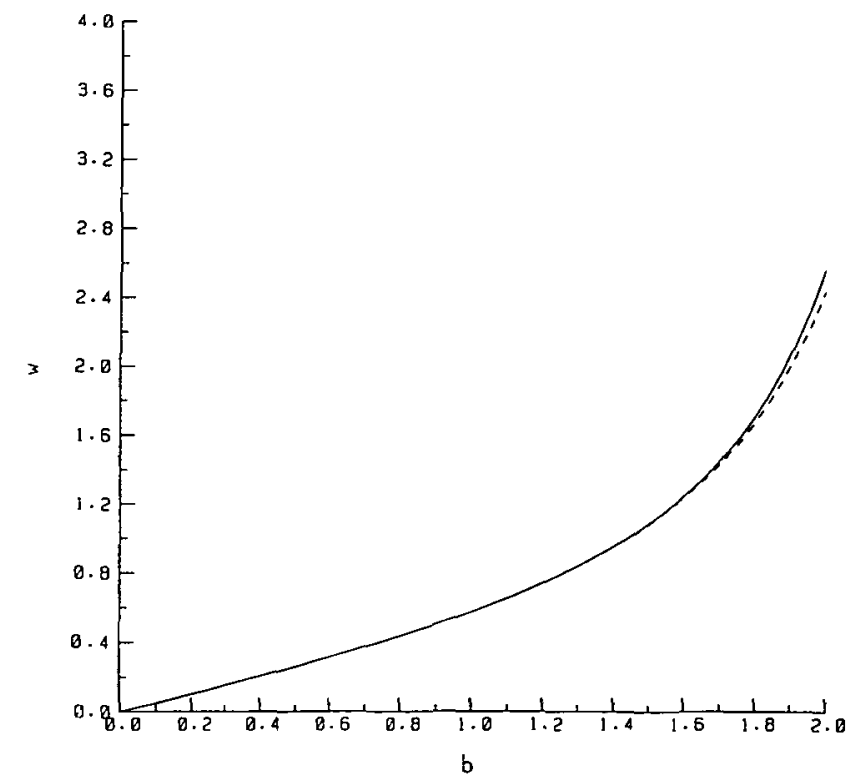

FIGURE 1. Comparison of exact values of $W,-$, and approximate values of $W, \ldots$ (a) one dimensional duct,

(b) a cylindrical tube. 
Here $\Sigma$ is the region in which the flame front propagates and $\partial \Sigma$ is the boundary of the region. Equation (3.10) is Helmholtz's equation with eigenvalue 1. Courant and Hilbert (1953) give that this eigenvalue is determined by the Rayleigh quotient

$$
1=\min _{\psi_{T}} \frac{W \int_{\partial \Sigma} \psi_{T}^{2} d S+\int_{\Sigma}\left(\underline{\nabla} \psi_{T}\right)^{2} d V}{\int_{\Sigma} \psi_{T}^{2} d V}
$$

where the minimum is taken over all functions $\psi_{T}$ satisfying the boundary condition (3.11). The Rayleigh quotient gives a simple method of estimating $W$ and hence estimating the correction $D_{2}$ to the Chapman-Jouguet velocity.

As an example consider the propagation of a flame front in a one-dimensional duct, for which the domain $\Sigma$ is the interval $-b \leq \xi \leq b$. A simple function $\psi_{T}$ which satisfies the boundary conditions (3.11) is

$$
\psi_{T}=A \xi^{2}-\frac{2 b A}{W}-A b^{2},
$$

where $A$ is a constant. From (3.12), we find that

$$
W=\frac{1-2 / 3 b^{2}-\left(1+14 / 45 b^{4}\right)^{1 / 2}}{b^{3} / 15-2 / 3 b}
$$

A comparison of this approximation to $W$ to the exact value given by (3.5) is shown in Figure 1(a). It can be seen that the approximation (3.14) is good even though the approximating function (3.13) is relatively simple.

The propagation of a flame front in a cylindrical tube, for which the domain $\Sigma$ is $0 \leq \rho \leq b, 0 \leq \theta \leq 2 \pi$, can be similarly treated. In this case, an approximating function $\psi_{T}$ satisfying the boundary condition (3.11) is

$$
\psi_{T}=A \rho^{2}-2 A b / W-A b^{2},
$$

$A$ being a constant. It can then be found from (3.12) that

$$
W=\frac{b^{2}-4+\left[16-b^{4} / 3\right]^{1 / 2}}{2 b-b^{2} / 3} \text {. }
$$

Figure 1(b) shows a comparison between this approximation to $W$ and the exact value given by (3.9). We again see that the approximation (3.16) is good even though the approximating function (3.15) is relatively crude. The Rayleigh quotient (3.12) should then give good approximations to $W$ for domains $\Sigma$ for which there are no analytic solutions of (3.10) and (3.11).

\section{Numerical solutions}

It was noted in section 2 that equation (2.6) governing the propagation of the front has the simple interpretation (2.22) that the front propagates along 
its normals at a speed dependent on the curvature. This observation allows a simple numerical solution of (2.6) to be developed. The numerical scheme described here is based on the scheme of Henshaw, Smyth and Schwendeman [4] which was developed for geometrical shock dynamics. In geometrical shock dynamics, a shock is propagated along its normals at a speed dependent on the local area (arclength in two dimensions) of the shock. The application of this method to the propagation of a flame fionit will now be outilined. Fuii details are given in Henshaw, Smyth and Schwendeman. In the present work, we shall consider two dimensional problems only.

Let us approximate the flame front by a discrete set of points $\underline{x}_{i}(t), i=$ $1, \ldots, N$ and let $K_{i}(t)$ and $\underline{n}_{i}(t)$ be the approximate front curvature and normal respectively at the point $\underline{x}_{i}(t)$. Then from (2.22)

$$
\frac{d}{d t} \underline{x}_{i}(t)=\left(D_{C J}-a K_{i}\right) \underline{n}_{i}(t) \quad i=2, \ldots, N-1 .
$$

This system of equations is solved using the two-step leap-frog scheme

$$
\underline{x}_{i}(t+\Delta t)=\underline{x}_{i}(t-\Delta t)+2 \Delta t\left(D_{C J}-a K_{i}(t)\right) \underline{n}_{i}(t), \quad i=2, \ldots, N-1
$$

where $\Delta t$ is the time step. This scheme is explicit and second order accurate in time. The positions of the points $\underline{x}_{1}$ and $\underline{x}_{N}$, which lie on the tube walls, are determined from those of $\underline{x}_{2}$ and $\underline{x}_{N-1}$ respectively so that the boundary condition (2.13) is satisfied.

To implement (4.2), both the approximate normal $\underline{n}_{i}(t)$ and approximate curvature $K_{i}(t)$ need to be determined. These are determined by differentiating two cubic splines to the data $\left(s_{j}(t), x_{j}(t)\right)$ and $\left(s_{j}(t), z_{j}(t)\right), j=1, \ldots, N$, where $s_{j}(t)$ is the discrete arclength given by

$$
s_{j}(t)= \begin{cases}0 & j=1 \\ s_{j-1}(t)+\left|\underline{x}_{j}(t)-\underline{x}_{j-1}(t)\right| & j=2, \ldots, N\end{cases}
$$

Let us denote these cubic spline interpolants by $\bar{x}(s)$ and $\bar{z}(s)$. The normal $\underline{n}_{i}(t)$ and curvature $K_{i}(t)$ are then given by

$$
\begin{gathered}
\underline{n}_{i}(t)=\frac{\left(\bar{z}^{\prime}\left(s_{i}\right),-\bar{x}^{\prime}\left(s_{i}\right)\right)}{\left[\left(\bar{x}^{\prime}\left(s_{i}\right)\right)^{2}+\left(\bar{z}^{\prime}\left(s_{i}\right)\right)^{2}\right]^{1 / 2}} \quad i=1, \ldots, N \\
K_{i}(t)=\frac{\bar{x}^{\prime}\left(s_{i}\right) \bar{z}^{\prime \prime}\left(s_{i}\right)-\bar{z}^{\prime}\left(s_{i}\right) \bar{x}^{\prime \prime}\left(s_{i}\right)}{\left[\left(\bar{x}^{\prime}\left(s_{i}\right)\right)^{2}+\left(\bar{z}^{\prime}\left(s_{i}\right)\right)^{2}\right]^{3 / 2}} \quad i=1, \ldots, N,
\end{gathered}
$$

where primes denote differentiation with respect to $s$.

While the front is started with a uniform point spacing at $t=0$, as the front propagates, the point spacing tends to become non-uniform. This is especially so when the region in which the front propagates is not straight. To maintain a fairly even point spacing, points are inserted in expansive 
regions and deleted in compressive regions of the front. This is done by checking the point spacing $\Delta s_{i}(t)$ periodically and requiring

$$
d_{1} \leq \frac{\Delta s_{i}(t)}{\Delta s_{\text {avg }}}=\sigma_{i}(t) \leq d_{2},
$$

where $d_{1}<1$ and $d_{2}>1$. If $\sigma_{i}(t)<d_{1}$, then the point is removed and if $\sigma_{i}(t)>d_{2}$, a new point $\underline{x}_{i-\frac{1}{2}}(t)$ is added using the cubic spline interpolant evaluated at $\frac{1}{2}\left(s_{i}(t)+s_{i-1}(t)\right)$. The values $d_{1}=0.25$ and $d_{2}=1.5$ were used for the numerical results presented in this section.

Two examples are considered in the present work; a flame front propagating in a channel with a hump and a flame front propagating around a $90^{\circ}$ circular bend. In both of these examples, the initial condition is taken to be a straight front and the boundary condition (2.13) is taken as

$$
\underline{n} \cdot \underline{\nabla} z_{S}=-1 \text {. }
$$

Also, the Chapman-Jouguet velocity $D_{C J}$ is taken to be 2 and the curvature coefficient 1.

Figure 2(a) shows a flame front propagating in a duct with a circular hump in it. It can be seen that the front settles to a steady state from the straight initial condition, is perturbed by the hump, then again settles to a steady state.
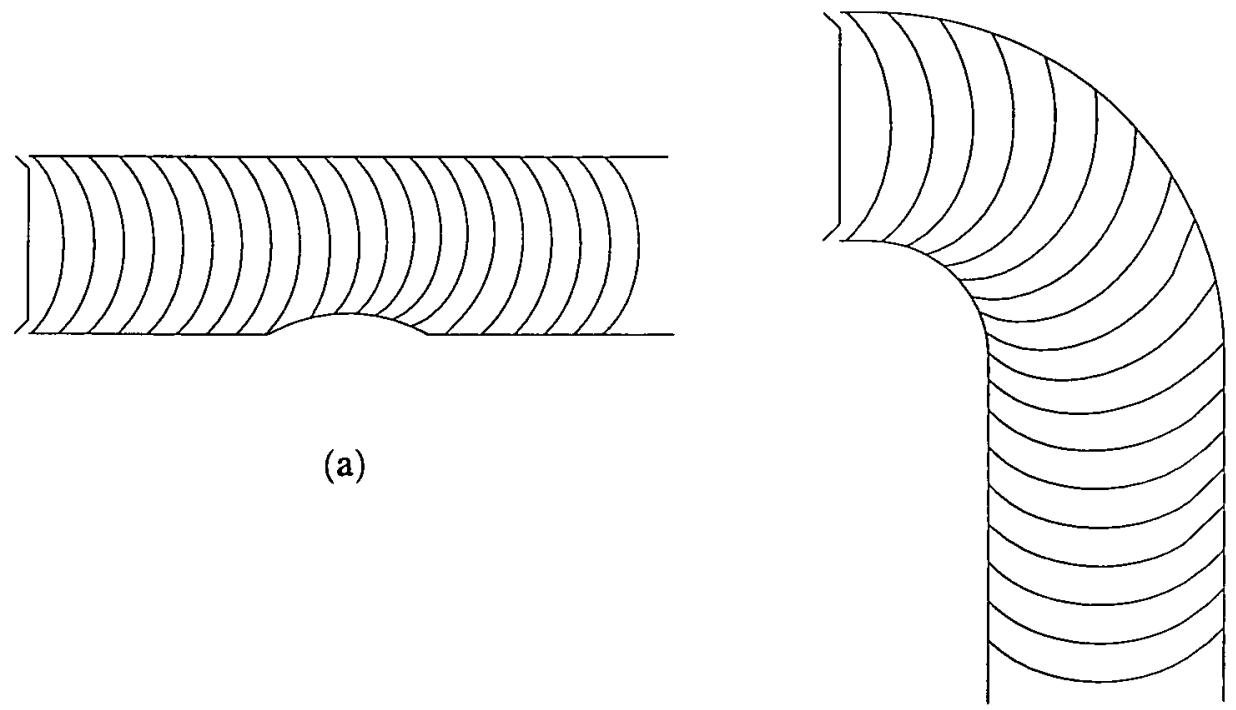

(b)

Figure 2. (a) Front in a duct of width 2 with a circular hump of height $2-\sqrt{3}$, subtending an angle of $60^{\circ}$.

(b) Front propagating around a $90^{\circ}$ circular bend of inner radius 1 and outer radius 3. 
Figure 2(b) shows a flame front propagating around a $90^{\circ}$ circular bend in a channel. The expansion of the front around the bend can be seen. Furthermore, it can be seen that the front settles to a steady state when it enters the straight portion after the bend.

The above examples are for two dimensional problems, and as such are rather limited in applicability. However, the numerical scheme can be extended to fully three dimensional problems. Propagating a flame front along its normals at a speed given by (2.22) is fast to perform numerically as it is a two dimensional problem in three dimensions. Solving the full set of equations (2.1) to (2.5) in three dimensions is a much more involved problem.

\section{References}

[1] J. B. Bdzil, "Steady-state two-dimensional detonation", J. Fluid Mech. 108 (1981) 195-226.

[2] E. Ben-Jacob, N. Goldenfeld, J. S. Langer and G. Schön, "Boundary- layer model of pattern formation in solidification," Phys. Rev. A 29(1) (1984) 330-340.

[3] R. Courant and D. Hilbert, Methods of Mathematical Physics, Vol. I (John Wiley and Sons, Inc., New York, 1953).

[4] W. D. Henshaw, N. F. Smyth and D. W. Schwendeman, "Numerical shock propagation using geometrical shock dynamics," J. Fluid Mech. 171 (1986) 519-545.

[5] J. W. Miles, "Diffraction of solitary waves”, Z. Agnew. Math. Phys. 28 (1977) 889-901.

[6] D. S. Stewart and J. B. Bdzil, “The shock dynamics of stable multidimensional detonation”, Comb. and Flame 72 (1988) 311-323.

[7] G. B. Whitham, "A new approach to problems of shock dynamics. Part I Two-dimensional problems", J. Fluid Mech. 2 (1957) 145-171.

[8] G. B. Whitham, "A new approach to problems of shock dynamics. Part II Three-dimensional problems", J. Fluid Mech. 5 (1959) 369-386.

[9] G. B. Whitham, Linear and Nonlinear Waves (J. Wiley and Sons Inc., New York, 1974). 